\title{
Una nueva expresión acerca del producto de convolución de la derivada de orden $\mathbf{k}$ de la delta de Dirac en $|x|-m^{2} \cdot *$
}

\author{
M. Aguirre. \\ Núcleo Consolidado Matemática Pura y Aplicada-NUCOMPA \\ Facultad de Ciencias Exactas UNCentro, Pinto 399, \\ 7000 Tandil, Argentina. \\ e-mail: maguirre@exa.unicen.edu.ar
}

(recibido/received: 16-Marzo-2010; aceptado/accepted: 16-Mayo-2010)

\section{RESUMEN}

En este artículo se obtiene una expansión en series (tipo Taylor) de la distribución $\delta^{(k)}\left(|x|^{2}-m^{2}\right)$, la cual permite dar una nueva expresión para el producto de convolución de $\left.\left\{\delta^{(k)}|x|^{2}-m^{2}\right)\right\} *\left\{\delta^{(t)}\left(|x|^{2}-m^{2}\right)\right\}$. Otras expresiones de este producto aparecen en ([5]).

Palabras claves: distribución, convolución, producto, delta de Dirac; teoría de distribuciones, AMS Subjet Classification, 46F10, 46F 12.

\section{ABSTRACT}

In this paper, we obtain a expansion in series (type Taylor) of distribution $\delta^{(k)}\left(|x|^{2}-m^{2}\right)$, and give a new expression for the convolution product of $\left.\left\{\delta^{(k)}|x|^{2}-m^{2}\right)\right\} *\left\{\delta^{(t)}\left(|x|^{2}-m^{2}\right)\right\}$. Other expressions of that product appear in ([5]).

Keywords: distribution, convolution, product, Dirac's delta function, theory of distributions, AMS Subjet Classification, $46 \mathrm{~F} 10,46 \mathrm{~F} 12$.

\footnotetext{
*Este trabajo es parcialmente soportado por la Comisión de Investigaciones Científicas de la provincia de Buenos Aires
} (C.I.C.), Argentina 


\section{INTRODUCCIÓN}

Sea $x=\left(x_{1}, \ldots x_{n}\right)$ un punto del espacio Euclideano $n$-dimensional $R^{n}$.

Consideremos las distribuciones $\left(|x|^{2}-m^{2}\right)_{+}^{\lambda} \mathrm{y}\left(|x|^{2}-m^{2}\right)_{-}^{\lambda}$ definidas por

$$
\left(|x|^{2}-m^{2}\right)_{+}^{\lambda}=\left\{\begin{array}{ccc}
\left(|x|^{2}-m^{2}\right)^{\lambda} & \text { si } & |x|^{2}-m^{2} \geq 0 \\
0 & \text { si } & |x|^{2}-m^{2}<0
\end{array}\right.
$$

y

$$
\left(|x|^{2}-m^{2}\right)_{-}^{\lambda}=\left\{\begin{array}{lll}
\left(-\left(|x|^{2}-m^{2}\right)\right)^{\lambda} & \text { if } & |x|^{2}-m^{2} \leq 0 \\
0 & \text { if } & |x|^{2}-m^{2}>0
\end{array}\right.
$$

donde $m$ es un número real positivo y $\lambda$ es un número complejo.

Las distribuciones $\delta^{(k)}\left(m^{2}-|x|^{2}\right)$ y $\delta^{(k)}\left(|x|^{2}-m^{2}\right)$ son definidas en ([5], página 341) en la siguiente forma:

$$
\delta^{(k)}\left(m^{2}-|x|^{2}\right)=\lim _{\alpha \rightarrow-k} \frac{\left(m^{2}-|x|^{2}\right)_{+}^{\alpha-1}}{\Gamma(\alpha)}
$$

y

$$
\delta^{(k)}\left(|x|^{2}-m^{2}\right)=(-1)^{k} \lim _{\alpha \rightarrow-k} \frac{\left(m^{2}-|x|^{2}\right)_{-}^{\alpha-1}}{\Gamma(\alpha)}
$$

donde $\Gamma(\alpha)$ es la función gama definida por:

$$
\Gamma(\alpha)=\int_{0}^{\infty} e^{-x} x^{\alpha-1} d x
$$

$\mathrm{y}$

$$
\operatorname{Re} s_{\alpha=-k} \Gamma(\alpha)=\frac{(-1)^{k}}{k !}
$$

([4], página 344).

Por otra parte, en este artículo necesitamos la siguiente fórmula:

$$
\left\{\delta^{(k)}\left(|x|^{2}-m^{2}\right)\right\}^{\wedge}=\frac{(-1)^{k} m^{n-2 k-2}}{2^{\frac{n}{2}}} \sum_{j \geq 0} \frac{\left(-|x|^{2}\right) m^{2 j}}{2^{2 j} j ! \Gamma\left(\frac{n}{2}+j-k\right)} \text { para } n \text { par si } k<\frac{n}{2}
$$




$$
\left\{\delta^{(k)}\left(|x|^{2}-m^{2}\right)\right\}^{\wedge}=\frac{(-1)^{k} m^{n-2 k-2}}{2^{\frac{n}{2}}} \sum_{j \geq 0} \frac{\left(-|y|^{2}\right)^{j} m^{2 j}}{2^{2 j} j ! \Gamma\left(\frac{n}{2}+j-k\right)} \text { para } n \text { par si } k \geq \frac{n}{2}
$$

([5], páginas 341-342).

Aquí el símbolo $\wedge$ designa la transformada de Fourier:

$$
\{f\}^{\wedge}=\int_{R^{n}} e^{<x, y>} f(x) d x
$$

$\mathrm{y}<x, y>=x_{1} y_{1}+x_{2} y_{2}+\ldots x_{n} y_{n}$

Ahora usando la fórmula

$$
\left\{\Delta^{j} \delta\right\}^{\wedge}=(-1)^{j}\left(|y|^{2}\right)^{j}
$$

([2]), página 201), donde $\Delta^{j}$ es el laplaciano iterado $j$ veces definido por

$$
\Delta^{j}=\left\{\frac{\partial^{2}}{\partial x_{1}^{2}}+\ldots \frac{\partial^{2}}{\partial x_{n}^{2}}\right\}^{j}
$$

$\mathrm{y}$

$$
|y|^{2}=y_{1}^{2}+\ldots y_{n}^{2}
$$

De (8) y (9), se tiene:

$$
\begin{gathered}
\left\{\delta^{(k)}\left(|x|^{2}-m^{2}\right)\right\}^{\wedge}=\frac{(-1)^{k} m^{n-2 k-2}}{2^{\frac{n}{2}}} \sum_{j \geq 0}\left\{\frac{\left(m^{2}\right)^{j} j^{j} \delta}{2^{2 j} j ! \Gamma\left(\frac{n}{2}+j-k\right)}\right\}^{\wedge} \\
\text { para } n \text { par si } k<\frac{n}{2}
\end{gathered}
$$

$\mathrm{y}$

$$
\left\{\delta^{(k)}\left(|x|^{2}-m^{2}\right)\right\}^{\wedge}=\frac{(-1)^{k} m^{n-2 k-2}}{2^{\frac{n}{2}}} \sum_{j \geq k-\frac{n}{2}+1}\left\{\frac{\left(m^{2}\right)^{j} \Delta^{j} \delta}{2^{2 j} j ! \Gamma\left(\frac{n}{2}+j-k\right)}\right\}^{\wedge}
$$

$$
\text { para } n \text { par si } k \geq \frac{n}{2} \text {. }
$$

De (13) y (14) se tiene,

$$
\left\{\delta^{(k)}\left(|x|^{2}-m^{2}\right)\right\}=\frac{(-1)^{k}}{2^{\frac{n}{2}}} \sum_{j \geq o}\left\{\frac{\left(m^{2}\right)^{j} \Delta^{j} \delta}{2^{2 j} j ! \Gamma\left(\frac{n}{2}+j-k\right)}\right\}
$$

$$
\text { para } n \text { par si } k<\frac{n}{2}
$$

$\mathrm{y}$

$$
\left\{\delta^{(k)}\left(|x|^{2}-m^{2}\right)\right\}=\frac{(-1)^{k}}{2^{\frac{n}{2}}} \cdot \sum_{l \geq k-\frac{n}{2}+1}\left\{\frac{\left(m^{2}\right)^{l} \Delta^{l-\frac{n}{2}+k+1} \delta}{4^{l-\frac{n}{2}+k+1} l !\left(l+k+1-\frac{n}{2}\right) !}\right\}
$$

para $n$ parsi $k \geq \frac{n}{2}+1$. 


\section{Aguirre}

Las fórmulas (15) y (16) aparecen en ([5]), página 74, formula (21) y (22) respectivamente.

Haciendo $m^{2}=0$ en (15) y (16) y usando la propiedad $\Delta^{0} \delta=\delta$, tenemos la siguiente fórmula:

$$
\begin{gathered}
\delta^{\left(\frac{n}{2}-1\right)}\left(|x|^{2}\right)=(-1)^{\frac{n}{2}-1} \pi^{\frac{n}{2}} \delta(x) \text { si } n \text { es par, } \\
\delta^{(k)}\left(|x|^{2}\right)=\frac{(-1)^{k} \pi^{\frac{n}{2}}}{\left(k-\frac{n}{2}+1\right) ! 4^{k-\frac{n}{2}+1}} \Delta^{k-\frac{n}{2}+1} \delta \text { si } n \text { es par y } k \geq \frac{n}{2}+1 .
\end{gathered}
$$

Usando que $\Delta^{0} \delta=\delta$ tenemos la siguiente fórmula:

$$
\delta^{(k)}\left(|x|^{2}\right)=\frac{(-1)^{k} \pi^{\frac{n}{2}}}{\left(k-\frac{n}{2}+1\right) ! 4^{k-\frac{n}{2}+1}} \Delta^{k-\frac{n}{2}+1} \delta \text { si } n \text { es par y } k \geq \frac{n}{2}-1
$$

donde $|x|^{2}=x_{1}^{2}+\ldots x_{n}^{2}$.

La fórmula (9), aparece en ([5], página 74, fórmula 24).

En este artículo, obtenemos una expansión en series (tipo Taylor) de la distribución $\delta^{(k)}\left(|x|^{2}-m^{2}\right)$ y se obtiene una nueva expresión para el producto de convolución de $\left\{\delta^{(k)}\left(|x|^{2}-m^{2}\right)\right\} *\left\{\delta^{(t)}\left(|x|^{2}-m^{2}\right)\right\}$. Otras expresiones para el producto de convolución aparecen en ([5]).

\section{EL PRODUCTO DE CONVOLUCIÓN $\left\{\delta^{(k)}\left(|x|^{2}-m^{2}\right)\right\} *\left\{\delta^{(t)}\left(|x|^{2}-m^{2}\right)\right\}$}

En este párrafo el símbolo * significa convolución.

Ahora, vamos a demostrar el siguiente lemma:

\section{Lemma 1:}

Sea $n$ la dimensión par del espacio y $k$ un entero no negativo entonces la siguiente fórmula es válida

$$
\delta^{(k)}\left(|x|^{2}-m^{2}\right)=\sum_{l \geq o} \frac{\left(-m^{2}\right)^{l}}{l !} \delta^{(k+l)}\left(|x|^{2}\right)
$$

La demostración del lemma 1 es consecuencia de la fórmula 16 y la fórmula 19. En efecto, haciendo $v=l-k+\frac{n}{2}-1$ en (16) y usando (19) se obtiene

$$
\begin{aligned}
& \left\{\delta^{(k)}\left(|x|^{2}-m^{2}\right)\right\}= \\
& =\frac{(-1)^{k}}{2^{\frac{n}{2}}} \sum_{l \geq 0} \frac{2^{\frac{n}{2}}\left(m^{2}\right)^{v}(-1)^{-k-v}\left(v-\frac{n}{2}+k+1\right) ! 4^{\nu-\frac{n}{2}+k+1} \delta^{(k+v)}\left(|x|^{2}\right)}{4^{v-\frac{n}{2}+k+1} v !\left(v-\frac{n}{2}+k+1\right) !}= \\
& \sum_{l \geq 0} \frac{\left(-m^{2}\right)^{v}}{v !} \delta^{(k+v)}\left(|x|^{2}\right) .
\end{aligned}
$$




\section{Aguirre}

Lemma 2:

Sea $n$ dimensión par del espacio, $k, t$ enteros no negativos tales que $k \geq \frac{n}{2}$ y $t \geq \frac{n}{2}$ entonces la siguiente fórmula es válida

$$
\begin{gathered}
\left\{\delta^{(k)}\left(|x|^{2}\right)\right\} *\left\{\delta^{(t)}\left(|x|^{2}\right)\right\}=(-1)^{\frac{n}{2}-1} \pi^{\frac{n}{2}} . \\
\frac{(k+t-n+2) !}{\left(k-\frac{n}{2}+1\right)\left(t-\frac{n}{2}+1\right) !} \delta^{\left(k+t-\frac{n}{2}+1\right)}\left(|x|^{2}\right) .
\end{gathered}
$$

Se sabe que ([5], página 75), la siguiente fórmula es válida

$$
\Delta^{t}\{\delta(x)\} \delta * \Delta^{s}\{\delta(x)\}=?^{t+s}\{\delta(x)\}
$$

para $t$ y $s$ enteros no negativos.

Ahora de (19) y usando (22) se tiene,

$$
\begin{aligned}
& \delta^{(k)}\left(|x|^{2}\right) * \delta^{(t)}\left(|x|^{2}\right)= \\
& \frac{(-1)^{k+t} \pi^{n}}{4^{k+l-n+2}\left(k-\frac{n}{2}+1\right) !\left(t-\frac{n}{2}+1\right) !}\left(\Delta^{k-\frac{n}{2}+1}\{\delta(x)\} \delta * \Delta^{t-\frac{n}{2}+1}\{\delta(x)\}\right)= \\
& \frac{(-1)^{k+t} \pi^{n}}{4^{k+l-n+2}\left(k-\frac{n}{2}+1\right) !\left(t-\frac{n}{2}+1\right) !} \Delta^{k+t-\frac{n}{2}+1-\frac{n}{2}+1}\{\delta(x)\}= \\
& \frac{(-1)^{\frac{n}{2}-1} \pi^{\frac{n}{2}}(k+t-n+2) !}{\left(k-\frac{n}{2}+1\right) !\left(t-\frac{n}{2}+1\right) !} \Delta^{\left(k+t-\frac{n}{2}+1\right)}\left(|x|^{2}\right)
\end{aligned}
$$

si $k \geq \frac{n}{2}-1$ y $t \geq \frac{n}{2}-1$.

De (23) se obtiene la fórmula (21).

Teorema:

Sea $n$ la dimensión par del espacio, $k, t$ enteros no negativos tales que $k \geq \frac{n}{2}$ y $t \geq \frac{n}{2}$ entonces la siguiente fórmula es válida

$$
\delta^{(k)}\left(m^{2}-|x|^{2}\right) * \delta^{(t)}\left(m^{2}-|x|^{2}\right)=\sum_{p \geq 0} A_{r, t, n, p} \delta^{\left(k+t+p-\frac{n}{2}+1\right)}\left(|x|^{2}\right)
$$

donde

$$
A_{r, t, n, p}=\frac{(-1)^{\frac{n}{2}-1} \pi^{\frac{n}{2}}(k+t+p-n+2) !}{p !\left(k-\frac{n}{2}+p+1\right) !\left(t-\frac{n}{2}+p+1\right) !}
$$

Demostración:

De (16) y considerando (23) se tiene, 


\section{Aguirre}

$$
\begin{aligned}
& \delta^{(k)}\left(m^{2}-|x|^{2}\right) * \delta^{(t)}\left(m^{2}-|x|^{2}\right)= \\
& \sum_{l \geq 0} \sum_{j \geq 0}{\frac{\left(-m^{2}\right)}{l ! j !}}^{l+j}\left[\delta^{(k+l)}\left(|x|^{2}\right) * \delta^{(t+j)}\left(|x|^{2}\right)\right]= \\
& (-1)^{\frac{n}{2}-1} \pi^{\frac{n}{2}} \sum_{p \geq 0}\left(-\frac{m^{2}}{4}\right)^{p}(k+t+p-n+2) ! \\
& \sum_{j=0}^{p} \frac{1}{j !(p-j) !\left(k+p-j-\frac{n}{2}+1\right) !\left(t+j-\frac{n}{2}+1\right) !} \delta^{\left(k+t+p-\frac{n}{2}+1\right)}\left(|x|^{2}\right) .
\end{aligned}
$$

Usando la fórmula

$$
\begin{aligned}
& \sum_{j=0}^{p} \frac{1}{j !(p-j) !\left(k+p-j-\frac{n}{2}+1\right) !\left(t+j-\frac{n}{2}+1\right) !}= \\
& \frac{1}{\left(k+p-\frac{n}{2}+1\right) !\left(t+p-\frac{n}{2}+1\right) !} \cdot \sum_{j=0}^{p}\left(\begin{array}{c}
k-\frac{n}{2}+1+p \\
j
\end{array}\right) \cdot\left(\begin{array}{c}
t-\frac{n}{2}+1+p \\
p-j
\end{array}\right)= \\
& \frac{1}{\left(k+p-\frac{n}{2}+1\right) !\left(t+p-\frac{n}{2}+1\right) !}\left(\begin{array}{c}
k-\frac{n}{2}+1+t-\frac{n}{2}+1+2 p \\
p
\end{array}\right)= \\
& \frac{(k+t-n+2 p+2) !}{\left(k+p-\frac{n}{2}+1\right) !\left(t+p-\frac{n}{2}+1\right) !} \cdot \frac{1}{p !(k+t-n+p+2) !},
\end{aligned}
$$

donde

$$
\left(\begin{array}{l}
t \\
j
\end{array}\right)=\frac{t !}{j !(t-j) !} .
$$

Reemplazando (27) en (26) se tiene,

$$
\begin{gathered}
\left\{\delta^{(k)}\left(|x|^{2}-m^{2}\right)\right\} *\left\{\delta^{(t)}\left(|x|^{2}-m^{2}\right)\right\}=(-1)^{\frac{n}{2}-1} \pi^{\frac{n}{2}} . \\
\sum_{p \geq 0} \frac{(k+t-n+2 p+2) !}{\left(k+p-\frac{n}{2}+1\right) !\left(t+p-\frac{n}{2}+1\right) !} \frac{\left(-m^{2}\right)^{p}}{p !} \delta^{\left(k+t+p-\frac{n}{2}+1\right)}\left(|x|^{2}\right)
\end{gathered}
$$

De (29) se concluye la demostración del teorema.

Es claro que haciendo $m^{2}=0$ en (24) bajo las condiciones $k \geq \frac{n}{2}-1, t \geq \frac{n}{2}-1$ y $n$ par, se obtiene la fórmula (23). 


\section{REFERENCIAS}

[1] Aguirre M. (1997) The distribution $\delta^{(k)}(P \pm i 0)$, Journal of Computational and Applied Math. (88) pp. 309-348, Elservier, N.H.

[2] L Gelfand, I. and Shilov, G. Generalized Functions, Vol. I, Academic Press, New York, 1964.

[3] Schwartz, L. Theorie the Distributions, Herman, Paris, 1966.

[4] Erdelchi, A. Higher Trascendental Functions, Vol. I and II, McGraw-Hill, New York, 1953.

[5] Erdelchi Aguirre T. Manuel., Distributional convolution product between the $k-t$ th derivative of Dirac's delta in $|x|^{2}-m^{2}$, Integral Transforms and Special Functions, 2000, vol. 10, No. 1, pp. 71-80.

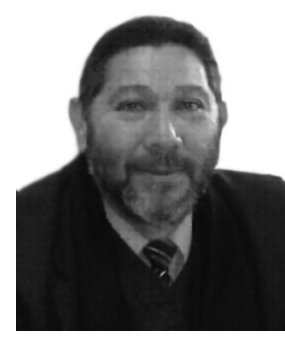

Manuel A. Aguirre, es Profesor y Decano de la Facultad de Ciencias Exactas de la Universidad Nacional del Centro de la Provincia de Buenos Aires, Núcleo Consolidado Matemática Pura y Aplicada-NUCOMPA

Facultad de Ciencias Exactas UNCentro

Paraje Arroyo Seco, 7000-Tandil

Provincia de Buenos Aires, Argentina

Tel.: +54 2293439657

E-mail: maguirre@exa.unicen.edu.ar 